\title{
Theory and Practice of Ideological and Political Teaching Methods of Professional Courses for Postgraduates
}

\section{— Based on the Course of Chemical Engineering Thermodynamics and Mass Transfer}

\author{
Ping Wang, Bo Dong ${ }^{*}$, Xi Wu, Luyuan Gong and Ying Li \\ School of Energy and Power Engineering, Dalian University of Technology, Dalian 116024, China \\ *Corresponding author. Email: bodong@dlut.edu.cn
}

\begin{abstract}
Guided by the integration of the teaching content system of Chemical Engineering Thermodynamics and Mass Transfer, a basic course for postgraduates, and the ideological and political content of the course, and for the purpose of solving the practical problems of ideological and political teaching and improving the teaching quality, this paper discusses the ideological and political teaching model and teaching methods of Chemical Engineering Thermodynamics and Mass Transfer, and explores the ideological and political education of postgraduate professional courses, carries out an effective way of ideological and political education curriculum. Chemical Engineering Thermodynamics and Mass Transfer is a course to train logical thinking. The abstraction and complexity in the process of analyzing problems often encounter many difficulties for postgraduate students who have just entered the campus. To guide students to truly understand and master concepts and make good use of them in engineering problems, the key is to make students clear their learning objectives, cultivate students' ability to overcome difficulties and solve practical problems, and enhance students' industry awareness and sense of social responsibility. Under the inquiry teaching mode, combining with the characteristic case teaching, selecting the knowledge points suitable for ideological and political education and infiltrating the moral education knowledge to students is an effective way to explore the ideological and political education of graduate professional courses and practice the ideological and political education curriculum.
\end{abstract}

Keywords: Chemical engineering thermodynamics, Mass transfer, Curriculum thought and politics, Teaching methods, Inquiry teaching mode.

\section{INTRODUCTION}

Chemical Engineering Thermodynamics is one of the most important compulsory courses for domestic and overseas chemical engineering and technology majors. It provides a theoretical basis for the operation and process design of various chemical units. It can solve engineering practical problems such as the calculation of thermodynamic properties in chemical processes, and gives the maximum utilization limit of substances and energy, which essentially guides how to slow down the rate of entropy increase and cultivates correct awareness of energy conservation and emission reduction from a scientific level. At the same time,
Chemical Engineering Thermodynamics is also a course to train logical thinking. It is abstract and complex in the process of analyzing problems. The knowledge system of Chemical Engineering Thermodynamics is deep and involves the derivation, differentiation, integration and other comprehensive calculations of advanced mathematics. The deduction and verification of thermodynamic formulas account for nearly $30 \%$ of the whole book [1]. For postgraduate students who have just entered the first grade; they often encounter many difficulties in learning, especially when analyzing practical problems with chemical engineering thermodynamics knowledge. During the process of teaching, if teachers only make a simple statement of 
the formula without corresponding expansion and application, it is difficult to explain the content of chemical engineering thermodynamics in a short class period. Therefore, how to grasp the essence of the course to guide students to truly understand and master concepts and make good use of them in engineering problems is the focus of thermodynamics teaching reform in recent years.

Mass transfer is a subject that studies the mass transfer process in material system. Its research plays an important role in deeply understanding some chemical process. The teaching task of mass transfer course is to enable students to understand and master the thermophysical properties and calculation of working medium, basic laws of mass transfer and calculation methods in energy and power engineering related technologies. The study plays an important role in cultivating students' deductive reasoning ability and the ability to use thermodynamic principles to solve practical engineering problems, and lays a necessary theoretical foundation for follow-up study, solving practical engineering problems and scientific research.

Chemical Engineering Thermodynamics and Mass Transfer is one of the compulsory courses for postgraduates in the school of energy and power engineering, and it is also one of the professional basic knowledge that postgraduates of energy and power engineering need to master and use flexibly. The basic task of this course is to study and master the thermodynamic properties and calculation of working medium in energy and environmental systems and related technologies by using the principles of classical thermodynamics and combining with the mathematical model reflecting system characteristics, so as to solve the problems such as the calculation of phase equilibrium and chemical equilibrium of industrial processes and mutual transformation and effective utilization of energy. Through the study of this course, students will learn to use the basic principles of thermodynamics to analyze engineering problems and deal with practical problems in energy and power engineering. It plays an important role in cultivating students' deductive reasoning ability and the ability to use thermodynamic principles to solve practical engineering problems, and lays a necessary theoretical foundation for the study of subsequent courses.

\section{EDUCATIONAL CONCEPT OF POSTGRADUATE PROFESSIONAL BASIC COURSES}

As a professional basic course for postgraduate students, the curriculum education concept of Chemical Engineering Thermodynamics and Mass Transfer is guided by the modern education concept. It organically integrates the elements of curriculum thought and politics, aims at cultivating high-quality innovative talents, integrates and improves the quality of teaching resources, speeds up postgraduate curriculum system and the modernization of teaching content, and constructs postgraduate students' autonomous and creative learning mode.Enhancing college students' ideological and political education with professional skills and knowledge has strong persuasion and appeal, which helps to maximize the function of the main channel of the class. It can also reverse the phenomenon of emphasizing intelligence over morality in professional curriculum teaching, which has irreplaceable advantages of other educational methods [2].

Curriculum ideological and political education is to decompose the ideological and political content into each course and give full play to the ideological and political education ability of each teacher. Infiltrating and enhancing ideological and political education in the curriculum makes students consciously strengthen their ideological and political cultivation and improve their political consciousness [3]. In the teaching process of Chemical Engineering Thermodynamics and Mass Transfer, a postgraduate professional basic course in the school of energy and power, it is very necessary to explore how to give full play to the educational function of professional basic course teaching, that is, to excavate the ideological and political elements in the course and explore how to carry out the ideological and political education of graduate professional courses.

\section{TEACHING METHOD DESIGN}

The process of curriculum ideological and political education should run through the whole process of curriculum teaching [4]. The use of inquiry teaching mode is conducive to cultivating students' participation and initiative in learning. The inquiry teaching mode refers to that in the teaching process, students are required to conduct independent learning, in-depth research and group cooperation and exchange on the main knowledge points in the current teaching content through the learning method characterized by "autonomy, exploration and cooperation" under the guidance of teachers, so as to better meet the requirements of cognitive goals and emotional goals in the curriculum standards. Inquiry teaching helps to promote students' learning and development, enhances students' learning initiative, and cultivates students' innovative ability and practical ability. Under the inquiry teaching mode, it is an effective method to select the knowledge points suitable for ideological and political education and infiltrate moral education knowledge to students. 


\subsection{Characteristic Case Teaching}

Firstly, the basic content of the course is designed into several cases. At the same time, considering the differences in the subject direction of postgraduates, students with similar research directions are encouraged to form groups. The contents arranged in the group discussion are as close as possible to the subject direction of postgraduates. For example, in their own research direction, students can introduce the contributions of Chinese scientists to the field of Chemical Engineering Thermodynamics and Mass Transfer and cultivate national pride. Students are encouraged to base themselves on the basic theory, pay attention to the international frontier, closely follow the development of scientific research and the needs of national construction in the new era, and promote the development of scientific research of Chemical Engineering Thermodynamics and Mass Transfer. This can not only improve students' learning enthusiasm, but also enable students to have a deeper understanding of the basic theory, and further apply the principle of thermodynamics to other courses and engineering practice. In teaching, we do not deliberately emphasize the deduction of formulas, but spend more time explaining the application of thermodynamic methods. In teaching, we should pay attention to the combination with engineering practice and integrate more closely with the forefront of discipline development. For example, Guocong Yu [5], a famous Chinese chemical distillation expert, developed a new way of threedimensional non-equilibrium simulation and amplification in terms of distillation theory and technology. Its distillation process and equipment is based on computational transfer. A relatively complete unsteady distillation theory and a new strategy of variable parameters of batch distillation and its corresponding technologies are also proposed, which also makes a contribution to the phenomenon of gasliquid two-phase interface and thermodynamics. Using his research results, a large number of distillation columns in petrochemical and chemical industry have been successfully transformed and remarkable results and huge economic benefits have been achieved. Under the influence of the scientific spirit of hard work and glory for the country of previous scientists, students have the courage to overcome difficulties in learning, which reflects the education of cultural self-confidence and national self-confidence.

\subsection{Expansion of Teaching Method}

As a core course, Chemical Engineering Thermodynamics and Mass Transfer has strong logic and reasoning. Using traditional teaching methods, it often takes a lot of time to repeat the content deduced from blackboard writing or PPT, which makes a lot of valuable class timecannot be effectively used, and the teaching progress is difficult to move forward. Finally, the teachers have to remove some practical content. Especially with the development of science and technology and the reform of teaching, there is more and more knowledge. Therefore, the class hours are relatively less and less. How to teach more content in less class hours and achieve better teaching effect? Modern teaching methods help solve this problem [6].

We designed appropriate teaching methods according to different teaching contents. For theoretical knowledge, we organize students to consult materials in combination with research topics to expand the amount of knowledge reading. For the application practice class, we organize students to use the Internet offline to learn the contents of specific experimental links involved in this course, and independently learn relevant courses through online resources such as the list of resource sharing courses in Chinese universities, so as to cultivate network information technology literacy. Through case teaching, the mature business software Aspen Plus is introduced into the practical application of teaching, so that students can focus on exploring solutions independently. For example, using Aspen Plus software to calculate the physical property data of liquefied natural gas multicomponent mixture is helpful to understand the thermodynamic properties. In the teaching process, teachers should not only teach students principles and formulas, but also teach them the background and scope of application of these principles and formulas, which is more important. In this way, when students apply chemical simulation and calculation software in their future work, they can correctly select the appropriate model to solve practical problems [7]. On the basis of perfecting the concept, the study of Chemical Engineering Thermodynamics and Mass Transfer should grasp three links: principlemodel-application. It emphasizes practicability and conciseness, take application as the purpose and pay attention to the cultivation of application ability [8].

\subsection{Design of Assessment System}

The course of Chemical Engineering Thermodynamics and Mass Transfer has many concepts, derivation formulas and strong theory. So it requires students to have a good mathematical foundation and abstract thinking ability. At the same time, teachers are required to adopt a variety of teaching methods and diversified assessment methods to cultivate students' interest in learning and improve students' learning initiative. The key is to make students clear their learning objectives, cultivate students' ability to overcome difficulties and solve practical problems, and enhance students' industry awareness and sense of social responsibility. Therefore, this course is very suitable for integrating ideological and political education. The examination method is one of the important links in the 
teaching reform of chemical thermodynamics. It is an effective method to check the implementation of the syllabus and evaluate the teaching quality. It is the main way to obtain the feedback information of teaching effect and an important basis for continuously improving and perfecting the whole teaching process [9].

According to the strong theoretical characteristics of Chemical Engineering Thermodynamics and Mass Transfer, the flexibility of the assessment system should be appropriately increased and diversified assessment should be emphasized. In addition to routine assessment links such as classroom attendance, questioning and final examination, the class group discussion after the end of each chapter is introduced with the help of the PPT report in class. At the same time, the design of the research topic should only focus on the students' research topics, so as to inspire students to pay attention to today's global economic development model and environmental protection issues, infiltrate the development status of energy and power engineering and energy shortage to students. It can also stimulate students' awareness of environmental protection and energy conservation.

\section{CONCLUSION}

In designing characteristic cases, expanding teaching means and applying diversified assessment system to the teaching link of Chemical Engineering Thermodynamics and Mass Transfer, we always grasp the primary task of ideological and political education in the course, excavate the ideological and political elements in the teaching link, reflect the synergy of peers in the same direction, and complete the ideological and political construction of the teaching course. This paper discusses the infiltration of moral education and ideological and political education in the course of Chemical Engineering Thermodynamics and Mass Transfer. In order to achieve the purpose of ideological and political education, teachers should find appropriate penetration points in this course, and moral education knowledge should be transmitted to students while imparting cultural knowledge. Ideological and moral education should run through one's life. The postgraduate stage is the most important stage of students' learning and life. Students have adapted to university campus life, their mentality tends to be calm, their thirst for knowledge is enhanced, and their selfmanagement ability is greatly improved. It is also a key period for students' growth. In this period they need positive guidance according to their psychological characteristics, so that students can improve their moral cultivation and establish a correct outlook on life, world outlook and values while learning scientific and cultural knowledge and skills.

\section{ACKNOWLEDGMENTS}

The work is supported by the "Education Reform Fund of the Graduate School of Dalian University of Technology (No. JG_2019057)".

\section{REFERENCES}

[1] Wei Long, Ying Xie, Yan Huang, Curriculum Reform of Chemical Thermodynamics under the Construction of High-level University of Science and Engineering, Chemical Industry Times, 2019, 33(7):46-48, 52.

[2] Deyi Gao, AidongZong, From Ideological and Political Course to Ideological and Political Education of Course: Constructing the Curriculum System of Ideological and Political Education in Colleges From a Strategic Height [J]. Higher Education, 2017 :(01)43-46.

[3] Daokun Lu, Some Core Problems and Solutions in the Implementation of Curriculum Thought and Politics-a Discussion Based on Professional Curriculum Thought and Politics[J].Ideological\& Theoretical Education, 2018,(03):64-69.

[4] Yufeng Shi, Yan Guo, Baosheng Zhang, Design and Practice of Ideological and Political Education in Engineering Thermodynamics [J]. Education Modernization, 2018, 5(40):290-291.

[5] Guozong $\mathrm{Yu}$, 360 Encyclopedias,https://baike.so.com/doc/66049356 818722.html, 2021-5-13

[6] Ling Ding, Yuhe Liang and Jun Han, LI Shiqian, Teaching Reform of Chemical Engineering Thermodynamics, Guangzhou Chemical Industry, 2010, 38(7):249-251.

[7] Xiaohua Lu, Xin Feng, Yuanhui Ji, Yanru Wang, Meeting the Second Spring of Chemical Engineering Thermodynamics [J].Higher Education in Chemical Engineering, 2008, 101(3):19-21.

[8] Cheli Wang, Cheli Wang, ZhaorongQiu, Jing Zhong, Jihe Yang, Ge $\mathrm{Xu}$, Construction and Integration of Core Curriculum Group of Chemical Engineering and Technology[J]. Higher Education in Chemical Engineering, 2005, 83(1):39-42.

[9] Yunpeng Zhao, Tao Jin, Minggang $\mathrm{Hu}$, et al, Research and practice on teaching reform of chemical thermodynamics, Chemical Industry Times, 2020, 34(6):40-42. 